\title{
ACOUSTOELECTRIC INVESTIGATION OF DEEP CENTERS IN BULK AND MULTILAYERED SEMICONDUCTORS
}

Two basic versions of the acoustic deep-level transient spectroscopy (A-DLTS) technique based on the acoustoelectric effect resulting from the interaction between an acoustic wave and interfaces have been used to study deep centers in semiconductor structures. The former uses the high frequency transverse acoustoelectric signal (TAS) arising from the interaction of surface acoustic wave electric field and free carriers at the structure interfaces. The latter uses an acoustoelectric response signal (ARS) produced by the structure interface when a longitudinal acoustic wave propagates through the structure. An additional version of A-DLTS uses the acoustoelectric effect on light beam generated interface in high resistivity and along with photosensitive semiconductors. Planar Si MIS structure and GaAs/AlGaAs heterostructure capacitors and high resistivity GaAs were investigated by these versions of the A-DLTS technique. Several deep centers were found and their activation energies and corresponding cross-sections determined. Both the appearance of some A-DLTS peaks and the shift of practically all peaks of the A-DLTS spectra with increasing bias voltage in investigated structures can be considered to be the characteristic features of interface states. The polarization and the propagation direction of acoustic waves generated on the light beam produced interface appears as important condition of detected deep centers.

\section{Introduction}

Semiconductors are the fundamental materials of the electronic industry because their properties can be manipulated over wide ranges through the control of impurities and other imperfections. While shallow impurities generally contribute extra charge carriers, electrons or holes and introduce minor perturbations in the crystal other impurities and a variety of lattice defects (vacancies, antisite defects, self-interstitials, etc.) constitute a more severe local perturbation, give rise to bound states that are considerably more localized, and often have energies deep in the band gap. We know all such impurities, lattice defects, and impurity-defect complexes as deep centers. Unlike shallow impurities the role of which is primarily to control the type and magnitude of conductivity, deep centers act primarily as carrier traps or recombination centers. The deep centers can control the lifetime of charge carriers and as such, deep centers are undesirable in devices where carriers must have long lifetimes, e.g., solar cells. On the other hand, they are useful when the carrier concentration needs to be reduced sharply on a short time scale, as in a fast switch. In addition, when the recombination or carrier-capture energy is released as light, deep centers are used in making light-emitting diodes (LEDs). Finally, many deep centers (vacancies, interstitials, vacancy-impurity complexes, etc.) play a major role in diffusion processes and in reactions that underlie materials modification (e.g., oxidation, recrystallization, etc.).

During the recent past, the semiconductor interfaces in metalsemiconductor contacts, semiconductor-insulator interfaces and semiconductor heterostructures are most important concepts in semiconductor devices and circuits that play a revolutionary role in microelectronics. New technologies, however require smaller devices, sharper transitions and higher number of preparation steps. Narrower transitions require sharper dopping profiles and subsequently lower temperatures during the preparation processes. Lower temperatures introduce defects that hardly can be removed by thermal treatment. These defects represent another new group of fabrication-induced defects.

The microscopic properties and the role of shallow impurities were already quite well understood through combination of theory and experiments in silicon and germanium. Deep centers on the other hand, proved far more difficult to investigate. Over the last thirty years, great progress has been achieved. Many experimental and theoretical techniques were developed and applied to particular systems. The choice of systems was dictated sometimes by technological concerns and sometimes by sheer academic curiosity. In many cases, as new techniques were developed, they were applied to systems that had been extensively studied with earlier techniques. The new information would either confirm, complement and expand existing knowledge about the center, or contradict it and thus lead to new understanding.

The interface deep states of semiconductor structures have been extensively studied and many useful experimental methods have been developed to characterize them [1 - 6]. Following the work of Lang [7], deep-level transient spectroscopy (DLTS) has become most powerful technique commonly used for the characterization of semiconductors and semiconductor structures because it reveals information about several characteristics of electrically or optically active defects present in such materials. Several useful variants of DLTS have been developed [8 - 11] and many attempts

\footnotetext{
* Peter Bury, Igor Jamnický, Peter Hockicko

Department of Physics, Faculty of Electrical Engineering, Žilina University, 01026 Žilina, Slovakia
} 
to improve the defect resolution capabilities of DLTS introducing different types of transient analysis procedure have been reported [12 - 17].

Recently, the acoustoelectric effect in semiconductor structures has been shown to be a useful tool for the experimental study of deep centers and two basic modifications of acoustic deep-level transient spectroscopy (A-DLTS) were introduced. The former surface acoustic wave (SAW) technique uses a nonlinear acoustoelectric interaction between the SAW electric field and the free carriers in an interface region which generates a transverse acoustoelectric signal (TAS) across the structure. Transient measurements of the rise or fall times of the resulting dc and hf part of the TAS have been used to study charged traps [18 - 21]. The latter longitudinal acoustic wave (LAW) technique uses an acoustoelectric response signal (ARS) observed at the interface of the semiconductor structure when a longitudinal acoustic wave propagates through the structure [21 - 23].

Both ARS and TAS are extremely sensitive to any changes in the space charge distribution in the interface region especially due to the trapped charge after an injection pulse has been applied. Their time development represents acoustoelectric transients, which reflect relaxation processes associated with the thermal recombination of excited carriers moving towards their equilibrium state Using a method of computer evaluation of isothermal acoustoelectric transients by applying a data compression algorithm [24] the activation energies and corresponding capture cross-sections can be determined from transient measurements of acoustoelectric response amplitudes as a technique of A-DLTS.

The technique of acoustic transient spectroscopy based on the utilization of space charge inhomogenity in high resistivity semiconductors produced by non-uniform illumination can be used to bulk deep centers investigation, too. The space charge inhomogenity can generate in proper conditions both the longitudinal and transversal or surface acoustic wave by applying a high frequency electric field [25]. The acoustoelectric transient technique consists then in the analysis of the amplitude time development of such generated acoustic wave after the light is turned off and that is detected by the receiving transducer. The trap states inside the semiconductor band gap are either filed or emptied by an optical illumination. After the external stimulus is removed, the traps either emit or capture charges to move forwards their equilibrium state.

In this contribution we present the principle of the A-DLTS technique as the acoustic spectroscopy technique using both SAW and LAW and describe the experimental procedures based on the computer- evaluation of isothermal acoustoelectric transients by applying a data compression algorithm and method of digital filtering by convolution. The A-DLTS technique has been applied for several kinds of semiconductor structures including $\mathrm{Si}$ and $\mathrm{GaAs}$ MIS structures, GaAs/AlGaAs heterostructures and high resistivity semiconductors to determine deep center parameters and proved to be an effective method to study deep centers that can play an important role in substrate materials used for electronic devices.

\section{Experimental principles and details}

The principle of A-DLTS techniques is based on the fact that the time development of the amplitude of the measured acoustoelectric signal (both TAS and ARS) after an injection pulse (electrical or optical) has been applied to the semiconductor structure ( $\mathrm{p}-\mathrm{n}$ junctions, MIS structures, heterostructures, ...) is proportion to the nonequilibrium carrier density, so that the decay time constant associated with the relaxation of the acoustoelectric signal amplitude is a direct measure of the time constant associated with the relaxation processes of injected carriers. The release of carriers from deep center levels that leads to the thermal equilibrium on a new steady state has the time dependence [19]

$$
\Delta n(t)=\Delta n_{t 0} e^{-\frac{t}{\tau}},
$$

where $\Delta n_{t 0}$ represents the variation in trap occupancy due to the acoustoelectric field and $\tau$ is the time constant associated with the release of the carrier from deep centers when injection pulse is turned off.

The ARS produced by semiconductor structure when a high frequency LAW propagates through the structure is proportional, as in the case of electromechanical capacitance transducer, to the voltage and relative change of capacitance induced by the acoustic wave. For the case of thin planar structure $(d<<\lambda)$ [22] is given by

$$
U_{a c}=\frac{Q}{C_{i}} \frac{p}{K_{i}}+\frac{Q}{C_{\omega}} \frac{p}{K_{\omega}}=\frac{Q}{C} \frac{p}{K},
$$

where in the case of MIS structure $C_{i}, C_{\omega}$ are the capacitances of insulator and space charge region and in the case of GaAs/AlGaAs heterostructure they are the capacitances of the insulating layer between two dimensional electron system (2-DES) and the electrode and the channel capacitance, respectively, $C$ is the total capacitance of the structure, $Q$ is the accumulated charge, $p$ is the acoustic pressure and $K$ is the elastic modulus assuming that elastic modules of the structure layers $K_{i}$ and $K_{\omega}$ are approximately equal. When a quiescent reverse bias voltage $U_{G}$ is applied to the MIS structure so that the structure is in deep depletion then for a short time a forward biased injection pulse is superimposed that results in filling of the interface states with majority carriers. After the filling pulse a new non-equilibrium depletion condition is established and due to the thermal emptying of the interface states the accumulated charge and simultaneously the capacitance of the structure is changed. As the ARS is able to reflect changes in the charge distribution in the interface region very sensitively the time development of the ARS after an injection bias pulse reflects relaxation processes associated with the thermally activated emission of excited carriers. From the acoustoelectric investigation of both MIS structures [22] and heterostructures [26] we can conclude that the ARS follows the accumulated charge behavior over the capacitance one.

By presenting the semiconductor structure capacitance as the equivalent capacitance of the series-connected capacitance of the dielectric and depletion layer in the case of MIS structure or the capacitance of insulating and channel layer in the case of 
$\mathrm{GaAs} / \mathrm{AlGaAs}$ heterostructure, the measured acoustoelectric response signal amplitude can be expressed then as follows

$$
U_{a c}^{0}(t)=U^{0} \exp (-t / \tau),
$$

Since this relation was obtained assuming only one deep center, the result can be readily generalized.

Non-destructive A-DLTS technique represented as SAW technique is based on the fact that the transversal acoustoelectric signal (TAS) arisen from the interaction of SAW electric field and free carriers in semiconductor structures reflect also any changes in the space charge distribution in the interface regions. The resulting electric field that develops across the structure can be expressed by the relation

$$
E=E_{0}+E_{1} e^{-i(\omega t-k x)},
$$

where $E_{0}$ is dc part and $E_{1}$ is hf part of TAS, respectively. Using hf part of TAS the acoustoelectric response signal is given by

$$
U_{a c}=\int_{0}^{d} E_{1} \sin (\omega t-k x) d x,
$$

where $d$ is the window width.

The special case occurs under the illumination of some part of high resistivity semiconductor by the weekly absorbed light of proper energy when free carriers are generated and the population of trapping centers in the illuminated part alters by the filling or emptying the deep center levels for electrons and holes. The nonequilibrium carriers then can immigrate from the illuminated to dark part of semiconductor and the space charge region arises producing the interface and internal electric field

$$
E_{s t}=-\frac{k T}{e\left(n_{0}+\Delta n\right)} \frac{d \Delta n}{d x},
$$

where $n_{0}$ is the equilibrium concentration of free carriers and $\Delta n$ is the concentration of excited electrons or holes. In piezoelectric semiconductor such as high resistivity GaAs, the sample being in the form of bar with its piezoactive direction orientated along the bar, the application of a hf electric field on the region with nonuniform distributed space charge can then lead to the periodic accumulation of electric charge that provides an additional hf electric field $[25,27,28]$ and generates an additional acoustic wave. After the illumination is turned off the photo-excited carriers recombine thereby establishing thermal equilibrium by the same way as in the previous cases so that the effect can be used to study deep centers in bulk semiconductors.

The present measurement technique compared with the original A-DLTS that uses the analysis of the acoustoelectric transient signal after an injection pulse similarly as in the Lang's DLTS developed for the capacitance transient by means of a set of emission rate windows [7], the present measurement technique is based on the computer-evaluated transients measured at fixed temperatures. The technique allows a single transient to be sampled at up to 8 different sample rates permitting 3 to 4 decades of time constants to be observed in one thermal scan. The differential ARS or TAS $\Delta U_{a c}$ can be then monitored as a function of temperature and peaks with maxim of the temperature for which the emission rate is the same as the adjusted sample rate. Because it is necessary to analyze only enough data to obtain the required information, specifically, the time constants of the transient at each temperature, a data compression algorithm was applied. For example, if 32767 data points are taken at a base sample rate $4 \mathrm{kHz}$, one could store the first 256 points, the extract another 256 points from the same transient by 128 averages of 2 points each, followed by extracting 512 points by 128 averages of 4 points each, etc. This storage scheme allows the transient to be observed at $4 \mathrm{kHz}$ for $64 \mathrm{~ms}, 2 \mathrm{kHz}$ for $128 \mathrm{~ms}$, $1 \mathrm{kHz}$ for $256 \mathrm{~ms}$ etc. up to $31.25 \mathrm{~Hz}$ for $8.192 \mathrm{~s}$. Thus many decades of time can be sampled from the same transient without permanently storing or processing redundant data. Sixteen transients were averaged per temperature. The temperature is then decreased to next temperature and the process is repeated. Additionally, the hard disk memory required to store such data does not need to exceed 2.3 kbytes per temperature comparing with 64 kbytes without data compression.

The software for the calculation has been thoroughly checked by performing a series of evaluations on computer-generated simulated transients. The purpose of this was to test the capability of the program to properly reveal the emission rates of the transients and the resolution of the method. The computer-evaluation of the observed isothermal ARS transients could be provided by both using Lang's original scheme [23] and correlation procedure with higher order on-line filters and rectangular weighing function [13].

Using the well known relation expressing the temperature dependence of the relaxation time characterizing the acoustoelectric transient [22]

$$
\frac{1}{\tau}=\sigma\left(v_{t h}\right\rangle N_{c} e^{-\frac{E_{T}}{k_{T}}},
$$

the activation energy, $E_{T}$, and corresponding capture cross-section, $\sigma$, can be determined. Here $\left\langle v_{t h}\right\rangle$ is the mean thermal velocity and $N_{c}$ the effective density of states at the bottom of the conduction band.

A block diagram of the experimental arrangement of the ADLTS techniques is shown in Fig. 1. The computer system was used to trigger the apparatus, to generate excitation pulses as well as to record and evaluate the isothermal transients of the acoustoelectric signals. A SAW of frequency $10 \mathrm{MHz}$ was generated using an interdigital transducer (IDT) evaporated on the $\mathrm{LiNbO}_{3}$ delay line and the structure to be investigated was placed on the top of the $\mathrm{LiNbO}_{3}$ and pressed against the window (detail-A). A LAW of frequency 4.6 or $13.2 \mathrm{MHz}$ was generated using a $\mathrm{LiNbO}_{3}$ transducer in the arrangement illustrated in detail-B. A fast and slow mode of shear acoustic wave or longitudinal acoustic wave of frequency $13 \mathrm{MHz}$ were generated on high resistivity GaAs through both the piezoactivity and additional acoustoelectric effect on space charge inhomogenity by applying hf electric field pulses along its $\langle 110\rangle$ direction and detected by shear and/or longitudinal quartz transducer (detail-C). The schematic illustration of the time arrange- 
ment of some experimental parameters corresponding to the isothermal transients scanning process is given in Fig. 2.

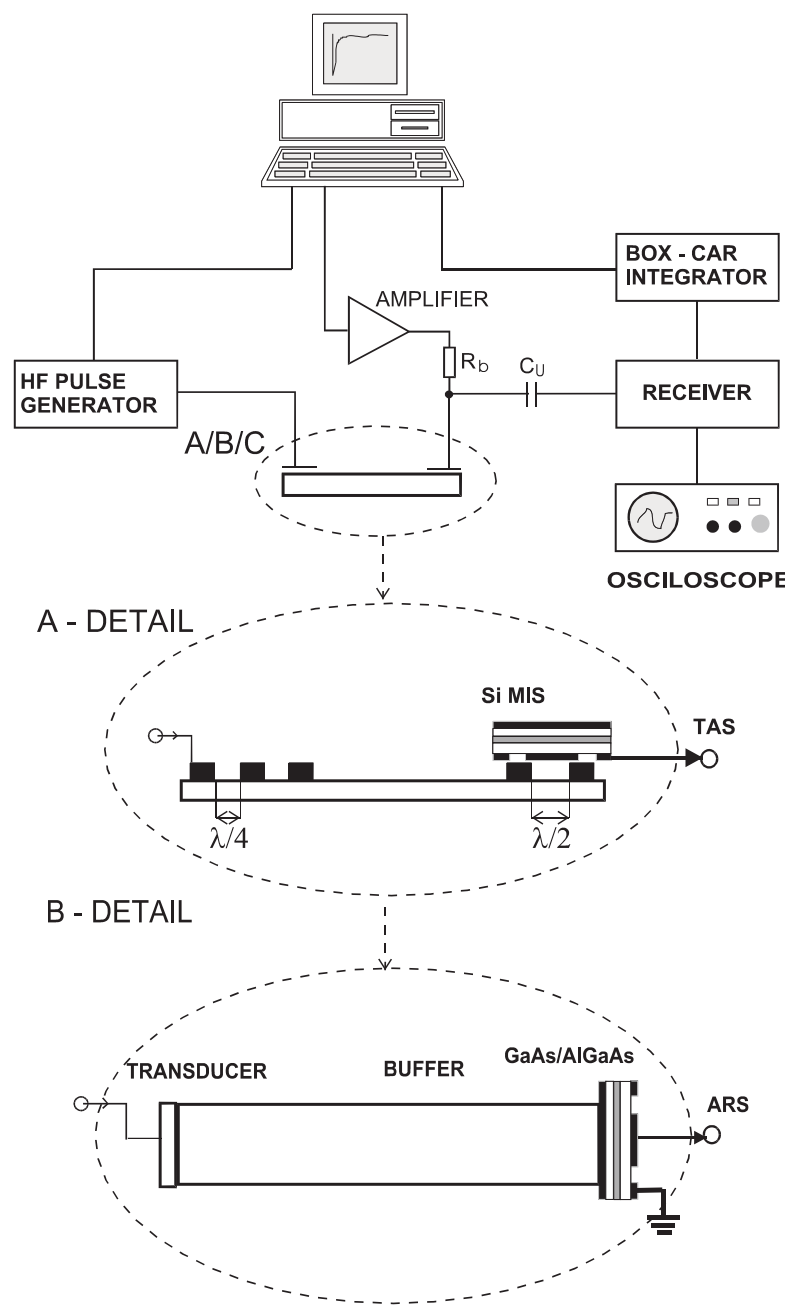

\section{C - DETAIL}

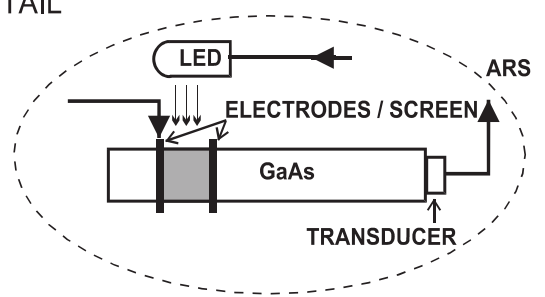

Fig. 1. Block diagram of the experimental arrangement for A-DLTS measurements. The detailed sample configurations are illustrated in A-detail (TAS measurement), B-detail (ARS measurement) and C-detail (illumination generated interface).

The both quiescent bias voltage pulse and illumination pulse with pulse width of $100-200 \mathrm{~ms}$ filling traps completely were applied to the structures and semiconductors, respectively using the computer with analogous - digital converter. The IR-LED with the maximum in spectral characteristic of $900 \mathrm{~nm}$ was used for the illumination The acoustoelectric signal produced by the structure after detection in the receiver was selected by the box-car integrator and then recorded and stored by computer.

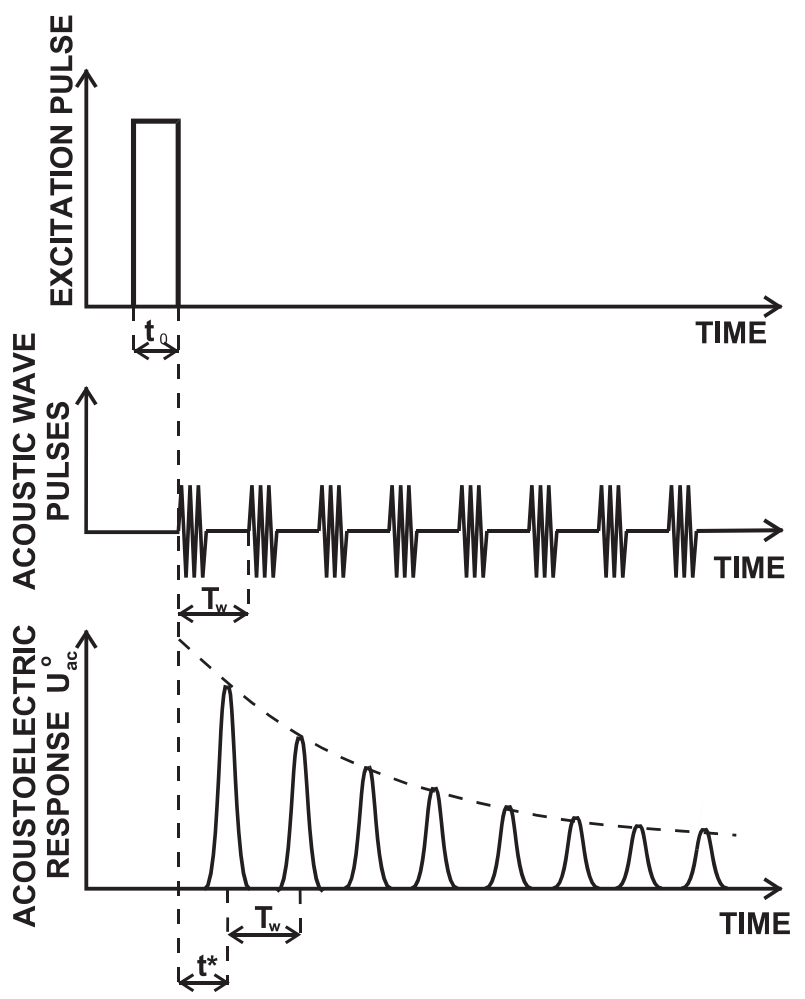

Fig. 2. Schematic illustration of the time arrangement of some experimental parameters, $t_{o}$ is the injection pulse width, $f_{w}=1 / T_{w}$ the rate frequency and $t^{+}$the transverse time through the buffer rod.

The well known Si MOS structures were firstly investigated to verify the principles of the above described method - Acoustic DLTS. The $\mathrm{Al}_{-} \mathrm{SiO}_{2}$-Si capacitors were fabricated both on n-type Si substrates with (100) surface orientation and 2.6 to $2.8 \Omega \mathrm{cm}$ resistivity and on p-type $\mathrm{Si}$ substrates with the same orientation and 8.0 to $8.7 \Omega \mathrm{cm}$ resistivity. The oxide layers were grown by CVD technique to thickness $80 \mathrm{~nm}$. Aluminum was deposited onto the insulator layer using a vacuum evaporation. Oxide from the backside of the wafer was removed and $\mathrm{Al}$ or $\mathrm{Ag}$ was deposited to provide the back contacts.

We have also investigated three different GaAs/AlGaAs heterostructures, which have been grown by MBE on semi-insulating (SI) GaAs substrates and prepared in the form of planar structure with two dimensional electron system - 2 DES (NU 169 and NU 1787) and two dimensional hole system - 2 DHS (NU 1323) at heterojunction. The first relatively simple investigated heterostructure (NU 169) consisted of the following layers: SI buffer layer, $2 \mu \mathrm{m}$; undoped AlGaAs spacer layer, $20 \mathrm{~nm}$; n-type doped AlGaAs 
layer with $1 \times 10^{18} \mathrm{~cm}^{-3} \mathrm{Si}, 40 \mathrm{~nm}$; SI GaAs capping layer, $20 \mathrm{~nm}$. The 2 DES was located at the interface between the SI GaAs layer and the AlGaAs spacer layer. Two ohmic contacts were made to reach the 2 DES layer. The schematic illustration of the heterostructure arrangement is in Fig. 3.

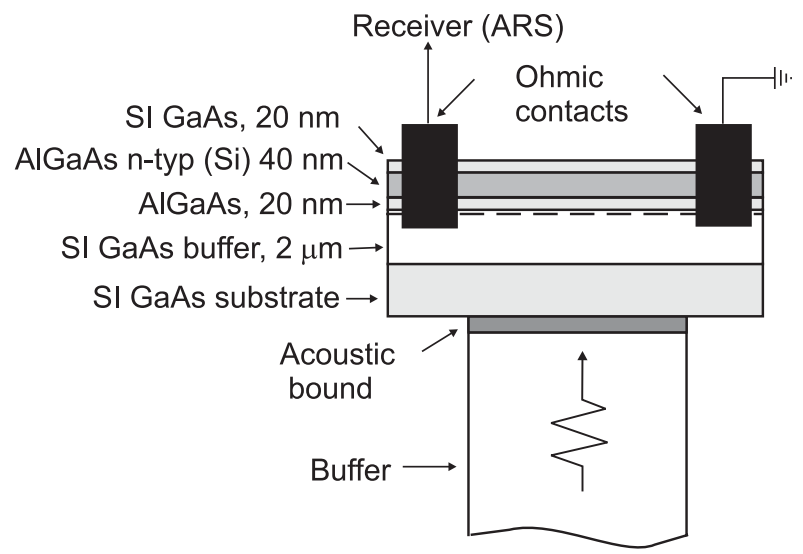

Fig. 3. Schematic illustration of experimental and layers arrangement of investigated GaAs/AlGaAs heterostructure (NU-169).

Another two heterostructures, NU 1787 and NU 41323 had similar layer structures consisting of superlattice. The layer structure of the sample NU 1787 consisted of the following layers: undoped GaAs, $1 \mu \mathrm{m}$; superlattice GaAs $(2.5 \mathrm{~nm}) / \mathrm{AlGa}(2.5 \mathrm{~nm})$, 50×; undoped GaAs, $0.5 \mu \mathrm{m}$; undoped AlGa, $50 \mathrm{~nm}$; n-type doped AlGa with $n=5.1017 \mathrm{~cm}^{-3}, 80 \mathrm{~nm}$; undoped GaAs, $17 \mathrm{~nm}$. The third heterostructure (NU 1323) with 2 DHS contained: undoped GaAs, $1 \mu \mathrm{m}$; superlattice $\mathrm{GaAs} / \mathrm{AlGa}, 5 \mathrm{~nm}(50 \times)$; undoped $\mathrm{GaAs}$, $0.5 \mu \mathrm{m}, \mathrm{AlGa}$ layer, $80 \mathrm{~nm}$; p-type doped AlGa layer with $n=$ $=5 \times 1017 \mathrm{~cm}^{-3}, 80 \mathrm{~nm}$; undoped GaAs, $17 \mathrm{~nm}$. The 2 DES and 2 DHS were located between undoped GaAs and AlGa layers.

The fast and slow mode of transversal acoustic wave $\left(T_{1}\right.$ and $T_{2}$ ) and longitudinal acoustic wave ( $\mathrm{L}$ ) of frequency $13 \mathrm{MHz}$ were generated through the acoustoelectric effect on space charge inhomogenity produced by illumination through the metal screen on high resistivity Cr-doped GaAs applying hf electric field pulses in $\langle 110\rangle$ direction and detected by transverse and longitudinal quartz transducers, respectively. The surface acoustic wave (SAW) of frequency $10 \mathrm{MHz}$ was generated in $\langle 001\rangle$ direction and detected by IDT evaporated directly on the sample rod.

\section{Results and Discussion}

Using the above-described techniques the development of the ARS as a function of temperature was for the first time investigated for Si MIS structures mentioned in the previous part. Fig. 4 shows typical ARS transients measured at various temperatures and the same bias voltage conditions for p-type Si MIS structure. These transients contain only one exponential component corresponding to $0.3 \mathrm{eV}$ trap. Fig. 5 represents a series of A-DLTS signals calculated from the isothermal ARS transients for various time constants (sampling times) using the correlation analysis with first order filter. Fig. 6 shows the series of A-DLTS spectra for different bias voltages applied to the Si MIS structure and the same time constant. As indicated by both the $C-U G$ and $U_{a c}-U_{G}$ measurements, by increasing $U_{G}$ towards negative values the $\mathrm{SiO}_{2}$ - Si interface region passes from deep depletion to nearly accumulation state. As it is shown in Fig. 6, peaks of the A-DLTS spectra are shifted to lower temperature with the increasing bias pulse voltage, that is characteristic features of the interface states [8].

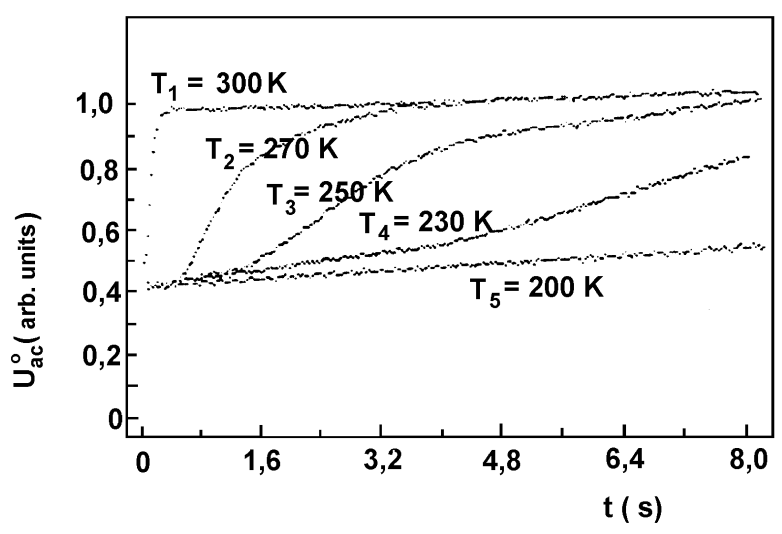

Fig. 4. Typical ARS transients recorded at various temperature.

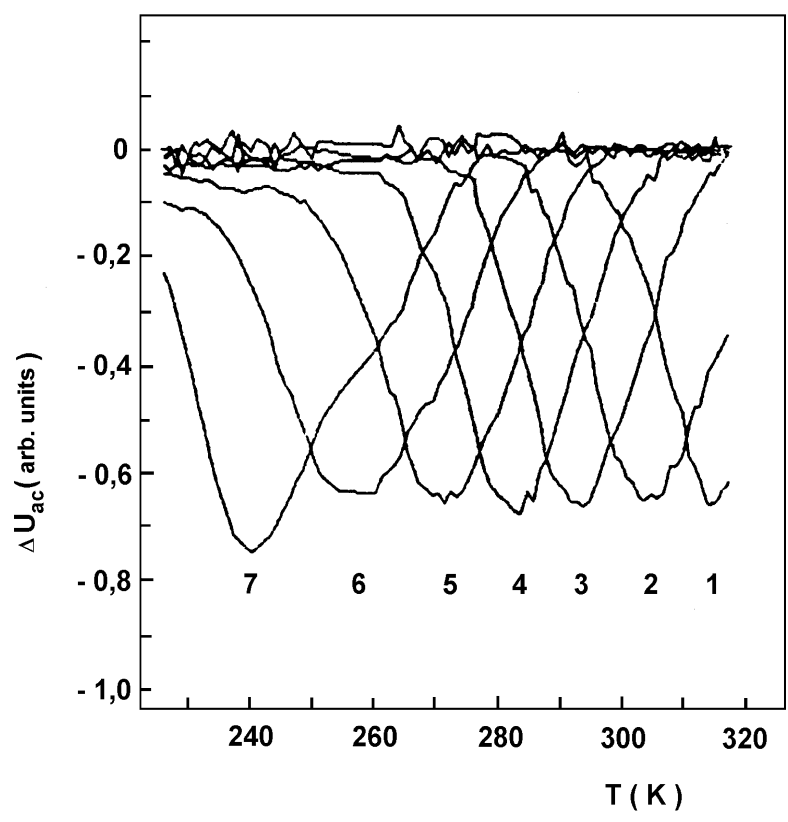

Fig. 5 A set of A-DLTS spectra calculated from isothermal transients for various relaxation times $\tau$, from $18,4 \mathrm{~ms}$ (1) to $1,178 \mathrm{~s}$ (7) measured at $U_{G}=4 \mathrm{~V}$.

The activation energies and corresponding capture cross-sections were determined from the Arrhenius plots (Fig. 7) constructed for the individual peaks from the A-DLTS spectra at different biases 
using the relation expressing the temperature dependence of the relaxation time characterizing the acoustoelectric transient. The obtained energy levels $0.47,0.30$ and $0.25 \mathrm{eV}$ above the valence band edge with the cross sections $4.8 \times 10^{-17}, 1.0 \times 10^{-19}$ and $1.5 \times 10^{-20} \mathrm{~cm}^{2}$ corresponding to the bias voltages 2,4 and $6 \mathrm{~V}$, respectively confirm the energy distribution of the interface states that is in a reasonable agreement with the many results found by DLTS and other techniques and are attributed to various defects $[5,6,29-31]$. The measurement in the inversion state $\left(U_{G}=9 \mathrm{~V}\right)$ showed the energy level $0.60 \mathrm{eV}$ with corresponding capture crosssection $4.8 \times 10^{-13} \mathrm{~cm}^{2}$. As the A-DLTS signal originates from the capture of electrons from the valence band into the trap level (hole emission process) and this appears as the negative signal in A-DLTS spectra [22], we believe that observed levels are deep acceptor levels.

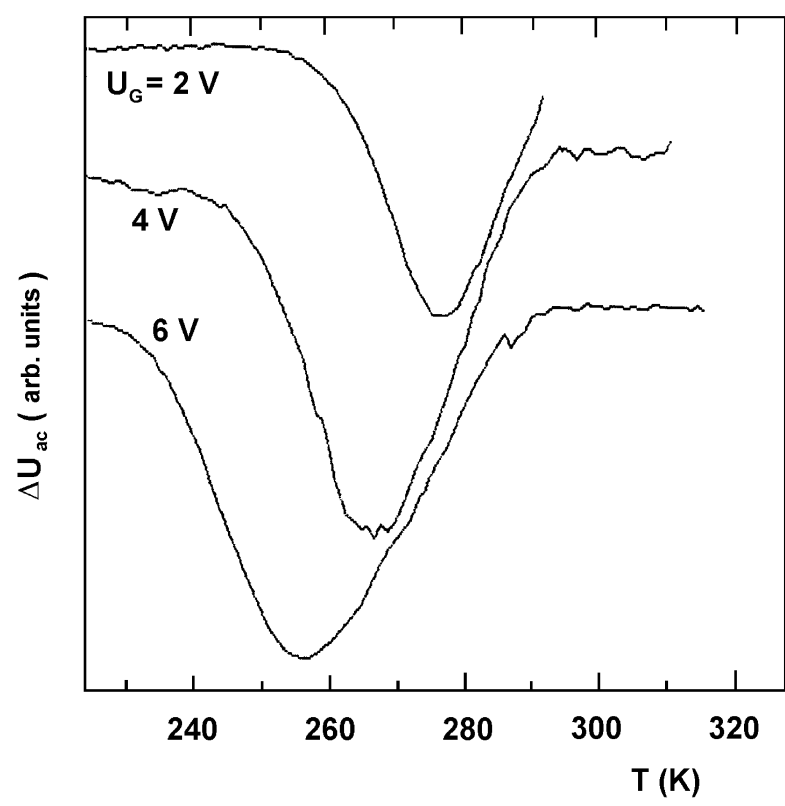

Fig. 6 A-DLTS spectra obtained from ARS transients as a function of bias voltage applied to the p-type Si MIS structure, $\tau=0.2945 \mathrm{~s}$.

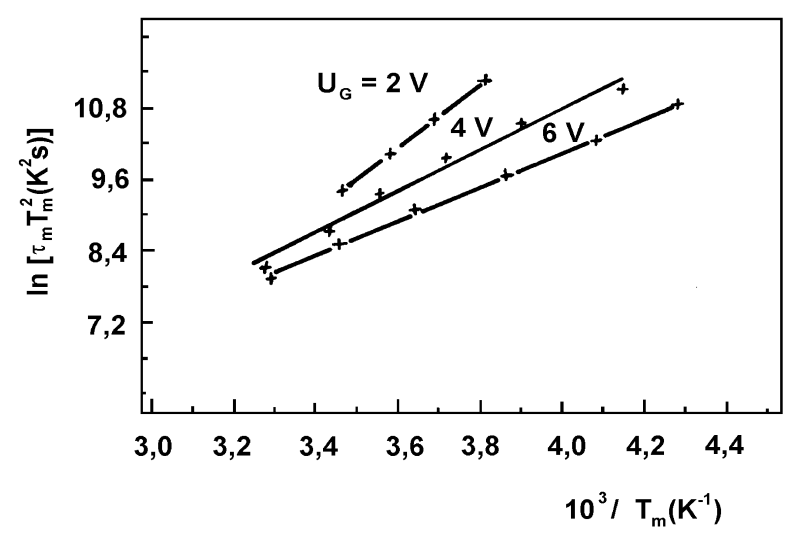

Fig. 7 Arrhenius plots constructed and calculated from the positions of the peak maxim using the A-DLTS spectra at different biases

$$
U_{G}=2,4 \text { and } 6 \mathrm{~V} \text {. }
$$

Fig. 8 represents a series of A-DLTS signals calculated from the isothermal acoustoelectric transients for various bias voltages applied to the n-type Si MIS structure. Both the $C-U_{G}$ and $U_{a c}-U_{G}$ measurements indicate that by increasing $U_{G}$ towards positive values the $\mathrm{SiO}_{2}$-Si interface region passes from the depletion state to the a state close to accumulation. The decreasing of

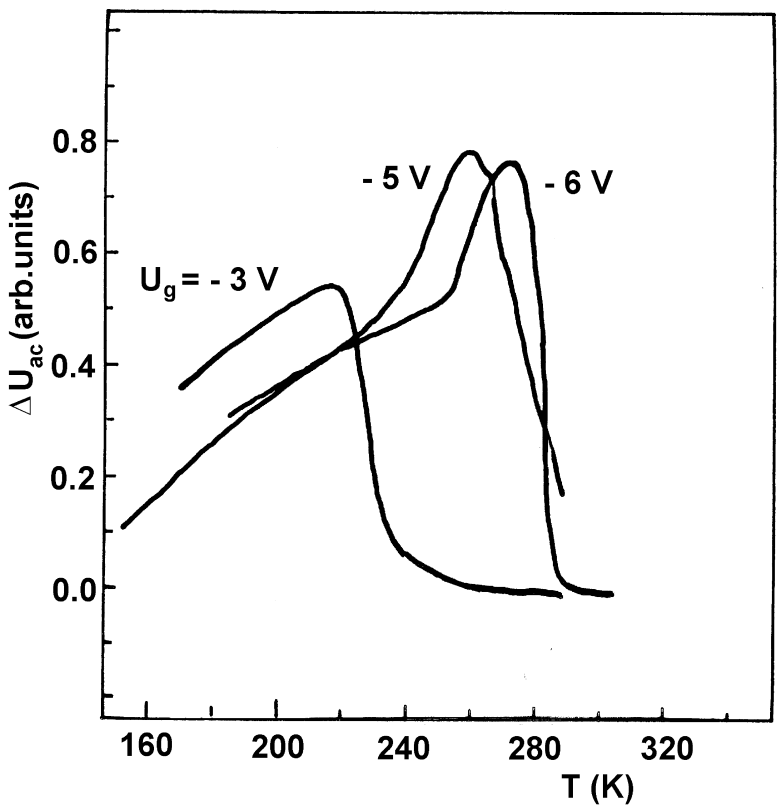

Fig. 8 A-DLTS spectra obtained from ARS transients as a function of bias voltage applied to the n-type Si MIS structure, $\tau=0,1472 \mathrm{~s}$.

UG under $-6 \mathrm{~V}$ indicated that an inversion state is created. The peaks of the A-DLTS spectra were shifted to lower temperature with the decreasing bias pulse voltage that again represents the characteristic features of the interface states. The obtained energy levels $0.52,0.59$ and $0.67 \mathrm{eV}$ and the cross sections $2.1 \times 10^{-15}$, $8.0 \times 10^{-14}$ and $4.6 \times 10^{-12} \mathrm{~cm}^{2}$ corresponding to bias voltages $-3,-5$ and $-6 \mathrm{~V}$, respectively, confirm the energy distribution of the interface states that is in a reasonable agreement with the results found by DLTS and other techniques $[6,32]$. In spite of the fact that the energy level position $0.54 \mathrm{eV}$ below the conduction band edge agrees with values mostly found for the bulk impurities and some structural defects it seems to be characteristic also for interface states. The measurements for the inversion state $\left(U_{G}=-9 \mathrm{~V}\right)$ showed the energy levels at $0.35 \mathrm{eV}$ and $0.32 \mathrm{eV}$ with corresponding capture cross sections $1.8 \times 10^{-17} \mathrm{~cm}^{2}$ and $5.9 \times 10^{-19} \mathrm{~cm}^{2}$, respectively. While the former energy level represents interface traps of donor character, the latter represents hole trap observed in the inversion layer [22].

Representative optically induced A-DLTS spectra of all investigated samples GaAs/AlGaAs with both 2 DES and 2 DHS obtained from acoustoelectric transients are illustrated in Fig. 9. The ADLTS spectrum of NU-169 sample contains three evident peaks representing three deep centers. The activation energies and corresponding capture cross-sections determined from the Arrhenius 
plots constructed for the individual peaks of A-DLTS spectra have the values: $0.29 \mathrm{eV}(1), 0.26 \mathrm{eV}(2), 0.21 \mathrm{eV}(3)$ and $2.9 \times 10^{-16}$ $\mathrm{cm}^{2}(1), 5.2 \times 10^{-16} \mathrm{~cm}^{2}(2), 1.8 \times 10^{-15} \mathrm{~cm}^{2}$ (3), respectively. While two of them, (1) and (2), have a donor character, the last center is of an acceptor type.

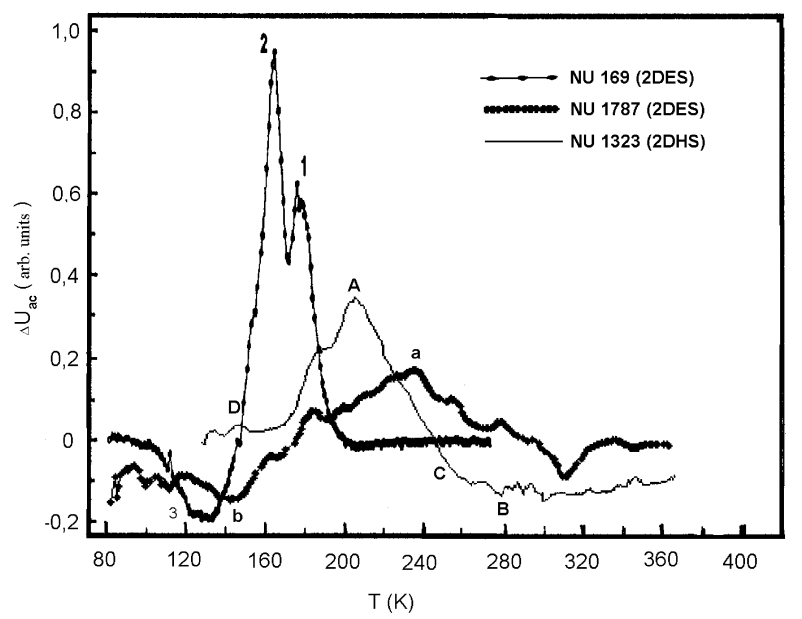

Fig. 9. A-DLTS spectra of investigated GaAs/AlGaAs heterostructures obtained for a relaxation time $\tau=0.0368 \mathrm{~s}$.

All obtained deep centers are well known from the measurements obtained by DLTS or other techniques, however never in the same composition. The deep centers with the energy level $0.29 \mathrm{eV}$ were detected in $\mathrm{GaAs} / \mathrm{AlGaAs}$ heterostructures [33], but also in GaAs layers prepared by MBE technique [34]. The deep center with energy $0.26 \mathrm{eV}$ is known also as DX center [35], sometimes with energies of $0.25-0.28 \mathrm{eV}$. Deep center of acceptor character with energy $0.21 \mathrm{eV}$ was detected in both $\mathrm{GaAs} / \mathrm{AlGaAs}$ or GaAs layers prepared by MBE technique [35, 36]. Some measurements [35] indicated even internal structure of obtained peaks like superposition of two different peaks similar as in our optically induced A-DLTS measurements [38].

The appearance of two broader peaks corresponding to deep centers of different type with some structure of smaller peaks is the characteristic feature of A-DLTS spectra obtained on NU 1787 sample containing also 2 DES. The activation energies of $0.29 \mathrm{eV}$ (a) and $0.10 \mathrm{eV}$ (b) with corresponding cross-sections of $2.2 \times 10^{-18} \mathrm{~cm}^{2}$ (a) and $5.5 \times 10^{-21} \mathrm{~cm}^{2}(\mathrm{~b})$, respectively were determined as parameters characterizing these deep centers. It should be noted that the A-DLTS signal at this case was much smaller (presented A-DLTS spectrum is even $\sim 2 \times$ amplified) comparing to previous spectrum and smaller peaks could not be easily used to deep centers characterization. The deep centers of acceptor type characterized by activation energy close to the value $0.1 \mathrm{eV}$ and not detected in previous sample were obtained in GaAs/AlGaAs heterostructures by SAW technique using dc TAS [18] and also by ordinary DLTS measurements [33].

The A-DLTS spectrum of NU-1323 sample with 2 DHS recorded by applying an optical injection pulse contains one dom- inant peak (A) and three weaker ones (B-D). Using Arrhenius plots, the following activation energies and corresponding capture cross-sections were determined: $1.29 \mathrm{eV}$ (A); $0.33 \mathrm{eV}$ (B); $0.73 \mathrm{eV}$ (C); $0.61 \mathrm{eV}$ (D) and $1.8 \times 10^{-19} \mathrm{~cm}^{2}$ (B); $9.2 \times 10^{-12} \mathrm{~cm}^{2}$ (C); $1.5 \times 10^{-12} \mathrm{~cm}^{2}(\mathrm{D})$, respectively. In spite the fact that some of the obtained deep centers (B-D) were registered also by other transient techniques using both electrical and optical pulse excitation $[33,36,37]$, they are typical for acoustical transient measurements $[2,10]$ and deep center $(\mathrm{A})$ characterized with relatively high activation energy, $1.29 \mathrm{eV}$ and large cross-section $\left(\sim 10^{-4}-10^{-6} \mathrm{~cm}^{2}\right)$ is known, except acoustic transient measurements [20, 28], only from TSC measurements [39].

As it can be seen the obtained values of activation energies are mostly in good agreement with the values found by both optically induced and other transient spectroscopy techniques and attributed to deep centers or other defects. The experimental arrangement indicates that detected deep centers should be localized close to the 2 DES or 2 DHS. However, some features found only by acoustic transient spectroscopy still remain unclear.

Fig. 10 and Fig. 11 show a typical A-DLTS spectra obtained for Si MIS structures and GaAs/AlGaAs heterostructures by nondestructive SAW A-DLTS technique, respectively. Using the above mentioned procedure, the activation energies and capture crosssections, summarized in Table 1, were calculated from the Arrhenius plots constructed for individual peaks of A-DLTS spectra.

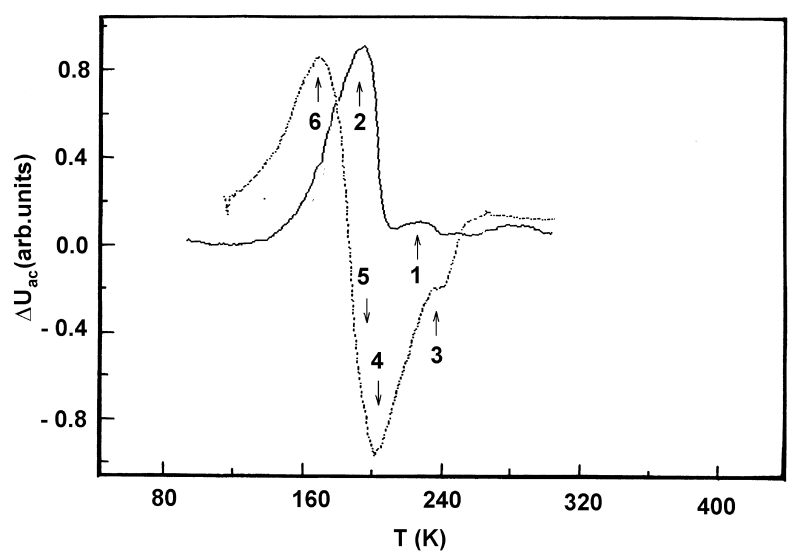

Fig. 10. A-DLTS spectra of $\mathrm{Si}(n)$ and $\mathrm{Si}(\mathrm{p})$ MIS structures obtained by $S A W$ technique.

The obtained values are mostly in good agreement with the values found by DLTS or others techniques [18, 24, 29, 30, 32, 40]. Comparing the presented results with those obtained by A-DLTS technique using longitudinal acoustic wave (Fig. 6 and Fig. 8) the energy levels are shifted because of the acoustoelectric transverse dc voltage is added to the excitation bias voltage.

Fig. 12. represents A-DLTS spectra of Cr-doped GaAs obtained for various generated acoustic waves (fast transversal $T_{1}$, slow transversal $T_{2}$, longitudinal $\mathrm{L}$ and SAW) and calculated from the isother- 
mal acoustoelectric transients. The activation energies and corresponding capture cross-sections determined from the Arrhenius plots are summarized in Table 2.

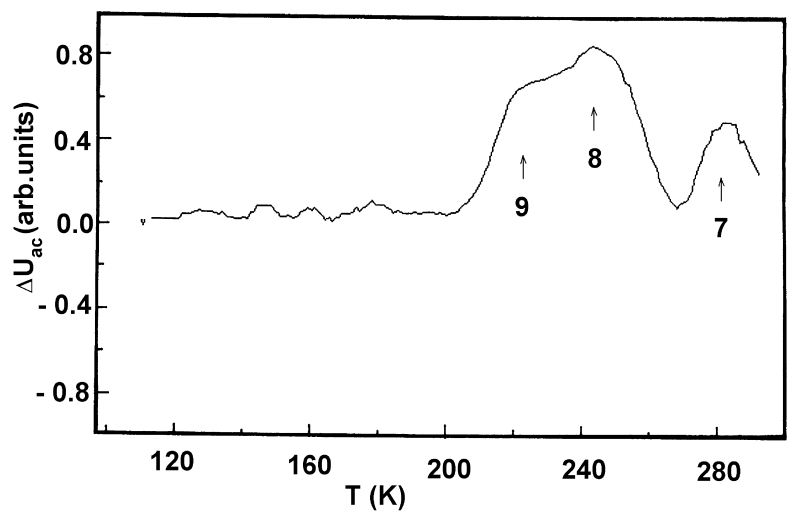

Fig. 11. A-DLTS spectra of GaAs/AlGaAs heterostructure obtained by SAW technique.

Summary of the deep centers parameters detected Table 1 by SAW A-DLTS in Si MIS and GaAs/AlGaAs heterostructures

\begin{tabular}{|c|c|c|c|c|}
\hline Label & Structure & $\mathrm{E}[\mathrm{eV}]$ & $\sigma\left[\mathrm{cm}^{2}\right]$ & $-U_{G}[\mathrm{~V}]$ \\
\hline 1 & $\mathrm{Si}(\mathrm{n}) \mathrm{MIS}$ & 0.37 & $7.8 \times 10^{-17}$ & 3.0 \\
\hline 2 & $\mathrm{Si}(\mathrm{n}) \mathrm{MIS}$ & 0.21 & $4.7 \times 10^{-19}$ & 3.0 \\
\hline 3 & $\mathrm{Si}(\mathrm{p}) \mathrm{MIS}$ & 0.49 & $3.3 \times 10^{-13}$ & 4.0 \\
\hline 4 & $\mathrm{Si}(\mathrm{p}) \mathrm{MIS}$ & 0.67 & $5.2 \times 10^{-11}$ & 4.0 \\
\hline 5 & $\mathrm{Si}(\mathrm{p}) \mathrm{MIS}$ & 0.47 & $6.5 \times 10^{-13}$ & 4.0 \\
\hline 6 & $\mathrm{Si}(\mathrm{p}) \mathrm{MIS}$ & 0.61 & $3.4 \times 10^{-6}$ & 4.0 \\
\hline 7 & $\mathrm{GaAs} / \mathrm{AlGaAs}$ & 0.47 & $1.3 \times 10^{-14}$ & 5.0 \\
\hline 8 & $\mathrm{GaAs} / \mathrm{AlGaAs}$ & 0.39 & $6.6 \times 10^{-16}$ & 5.0 \\
\hline 9 & $\mathrm{GaAs} / \mathrm{AlGaAs}$ & 0.67 & $2.7 \times 10^{-12}$ & 7.0 \\
\hline
\end{tabular}

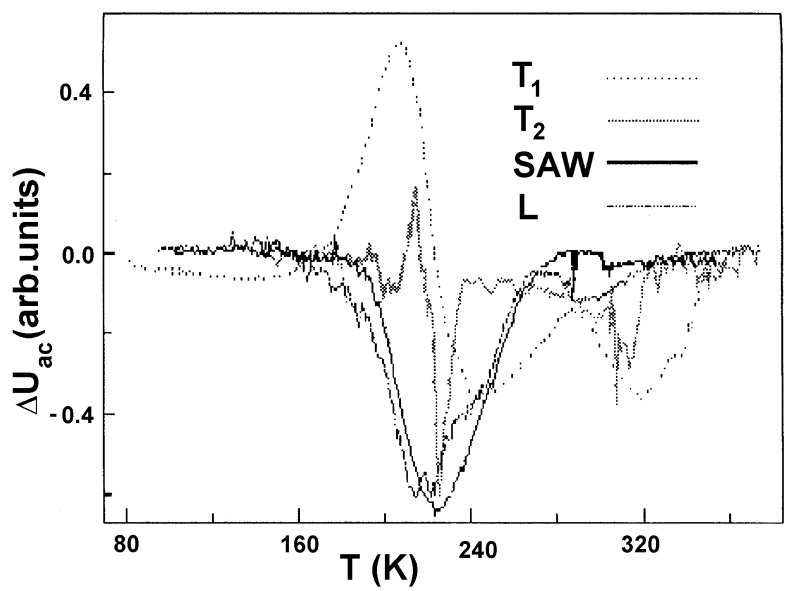

Fig. 12. Optically induced A-DLTS spectra of Cr-doped GaAs obtained for various acoustic waves (fast transversal $T_{1}$, slow transversal $T_{2}$, longitudinal $L$ and $S A W$ )
Summary of the centers parameter detected

Table 2

in high resistivity GaAs for various acoustic waves

\begin{tabular}{|l|l|c|c|}
\hline Propagation & Polarization & \multicolumn{2}{|c|}{ Deep Centers } \\
\cline { 3 - 4 } Direction & & $\mathrm{E}[\mathrm{eV}]$ & $\sigma\left[\mathrm{cm}^{2}\right]$ \\
\hline$[110]$ & {$[001](\mathrm{T} 1)$} & 1.28 & $3.1 \times 10^{-4}$ \\
& & 0.72 & $2.4 \times 10^{-13}$ \\
& & 0.47 & $3.0 \times 10^{-15}$ \\
& & 0.31 & $4.8 \times 10^{-18}$ \\
& & 0.27 & $2.1 \times 10^{-18}$ \\
& & 0.35 & $1.7 \times 10^{-16}$ \\
& & 0.22 & $4.1 \times 10^{-18}$ \\
\hline$[110]$ & & 1.10 & $1.6 \times 10^{-6}$ \\
& & 0.79 & $1.1 \times 10^{-11}$ \\
\hline$[110]$ & {$[110]\left(\mathrm{T}_{2}\right)$} & 0.74 & $5.1 \times 10^{-12}$ \\
& & 0.32 & $2.0 \times 10^{-7}$ \\
\hline$[001]$ & {$[110](\mathrm{L})$} & 0.77 & $2.5 \times 10^{-17}$ \\
\hline
\end{tabular}

Most of the obtained energy levels of deep centers are in good agreement with the values found by the other techniques [36, 37, 42] that have been already compared and discussed [41].

The comparison of the results obtained for different acoustic waves $\left(T_{1}, T_{2}\right.$, L, SAW) generated on the same sample and mostly for the same screen indicates that the interaction of acoustic wave and deep centers depends both on the propagation direction and on the polarization of generated acoustic wave.

\section{Conclusion}

In conclusion, the acoustoelectric investigation using the acoustic transient spectroscopy technique represented by several various versions we presented can be successfully used to study the deep centers in semiconductors and semiconductors structures. Several deep centers attributed to the interface states in Si MIS structures, GaAs/AlGaAs heterostructures with both 2 DES and 2 DHS and high resistivity GaAs were discovered and their parameters were determined.

The presented and for deep centers investigation used acoustoelectric transient technique induced also some advantages comparing with the other transient techniques: the acoustoelectric signal is produced directly by a heterojunction containing space charge so that any changes in its distribution are immediately reflected by the ARS and/or TAS; the number and thickness of individual insulating layers at the heterostructure does not influence obtained acoustoelectric response; the quality of the ohmic contacts does not play so important role as in electric techniques.

Acknowledgements The authors would like to thank Dr. O Mikuš (MIS structure) and Dr. M. Henini (GaAs/AlGaAs heterostructures) for the growing of the layers and Mr. F. Černobila for technical assistance. This work was partly supported by Grant No.1/8308/01 of the Slovak Ministry of Education. 


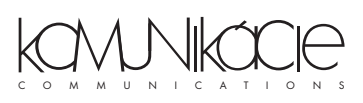

\section{References}

[1] TERMAN, L.: Solid State Electronics 5, (1962) 285.

[2] NiCOllian, E. H., GOetZBERGER, A., Berglund, C. N.: Appl. Phys. Letters 15 (1969) 174.

[3] YOUNG, D. R., IRENE DI MARIA, E. A., De KEERSMAECKER, R. F.: J. Appl. Phys. 50 (1979) 6366.

[4] TSENG, W. L.: J. Appl. Phys. 62 (1987) 591.

[5] PATER, K.: Appl. Phys. A 44 (1987) 191.

[6] HUNG, K. K., CHENG, Y. C.: J. Appl. Phys. 62 (1987) 4204.

[7] LANG, D. V.: J. Appl. Phys. 45, (1974) 3023.

[8] YAMASAKI, K., YOSHIDA, M., SUGANO, T.: J. Appl. Phys. 18 (1979), Japan, 113.

[9] KIROV, K. I., RADEV, K. B.: Phys. Stat. Sol. (a) 63 (1981) 711.

[10] TURChANIKOV, V. I., LYSENKO, V. S., GUSEV, V. A.: Phys. Stat. Sol. (a) 95 (1986) 283.

[11] SCHUlZ, M., JOHNSON, N. M.: Appl. Phys. Letters 31 (1977) 622.

[12] DOOLITTLE, W. A., ROHATGI, A.: Rev. Sci. Instrum 63 (1992) 5733.

[13] CROWELL, C. R., ALIPANAHI, S.: Solid St. Electron. 24 (1981) 25.

[14] WANG, C. W., WU, C. H., BOONE, B. L.: J. Appl. Phys. 73 (1993) 760.

[15] DOBACZEWSKI, L., KACZOR, P., HAWKINS, I. D., PEAKER, A. R.: Appl. Phys. 76 (1994) 194.

[16] DMOWSKI, K.: Solid State Electronics 38 (1995) 1051.

[17] THURZO, I., GMUCOVÁ, K.: Rev. Sci. Instrum 65 (1994) 2244.

[18] TABIB-AZAR, M., HAJJAR, F.: IEEE Transactions on Electron Devices 36 (1989) 1189-1195.

[19] ABBATE, A., MAN, K. J., OSTROVSKIJ, I. V., DAS, P.: Solid State Electronics 36 (1993) 697.

[20] BURY, P., JAMNICKÝ, I.: Proc. 16th Int. Congr. on Acoustics, Vol. I, Seattle, (1998) 429.

[21] BURY, P., JAMNICKÝ, I., RAMPTON, V. W.: Physica B 263-264 (1999) 94.

[22] BURY, P., JAMNICKÝ, I., ĎURČEK, J.: Phys. Stat. Sol. (a) 126 (1991) 151.

[23] JAMNICKÝ, I., BURY, P.: Phys. Stat. Sol. (a) 139 (1993) K35.

[24] BURY, P., JAMNICKÝ, I.: Acta Phys. Slovaca 46 (1996) 693.

[25] BURY, P., HOCKICKO, P., JAMNICKÝ, I.: Proc. 17th Int. Congress on Acoustics, Rome, Vol. I, 2001.

[26] BURY, P., RAMPTON, V. W., CARTER, P. J. A., McENANEY, K. B.: Phys. Stat. Sol. (a) 133 (1992) 363.

[27] ŠTELINA, J., BRACINÍK, J.: Appl. Acoustic 44, (1995) 369.

[28] BURY, P.: Proc. 16th Int. Congr. on Acoustics, Vol. I, Seattle, (1998) 431.

[29] KAR, S., NARASIMHAN, R. L.: J. Appl. Phys. 61, (1987) 5353.

[30] DAI, Z.: Solid State Electronics 32, (1989) 439.

[31] McLARTY, P. K., COLE, J. W., GALlOWAY, K. F., IOUNNOU, D. E., BERNACKI, S. E.: Appl. Phys. or 13.2 MHz was Letters 51, (1989) 1087.

[32] HOFMAN, F., KRAUTSCHNEIDER, W. H.: J. Appl. Phys. 63, (1989) 1358-1360.

[33] AS, D. J., EPERLEIN, P. W., MOONEY, P. M.: J. Appl. Phys. 64 (1988) 2408.

[34] WANG, C. W., WU, C. H.: J. Appl. Phys. 74 (1993) 3921.

[35] ENRIQUEZ, L., DUENAS, S., BARBOLA, J., IZPURA, I., MUŇOZ, E.: J. Appl. Phys. 72 (1992) 525.

[36] DARMO, J., DUBECKÝ, F., KORDOŠ, P., FORSSTER, A.: J. 393 Luth, Mat. Science B 28 (1994) 4393.

[37] MARTIN, G. M., MITONNEAU, A., MICREA, A.: Electr. Lett. 13 (1977) 193.

[38] BURY, P., HOCKICKO, P., RAMPTON, V. W.: Acta Phys. Slovaca (will be published).

[39] LIN, A. L., BUBE, R. H.: J. Appl. Phys. 47 (1976) 1859.

[40] OKUMURA, M., MISAWA, S., YOSHIDA, S.: Surface Science 174 (1986) 324-330.

[41] BURY, P., JAMNICKÝ, I.: World Congress on Ultrasonics, Berlin (1995) 535.

[42] BURY, P.: Proc. 16th Int. Congress on Acoustics, Vol. I, Seattle (1998) 431.

[43] DUBECKÝ, F., SAFRÁNKOVÁ, J., OLEJNÍKOVÁ, B.: Crystal Properties \& Preparation 12 (1987) 147-152. 